\title{
Editorial: Immunological Mechanisms, Biomarkers and Immunotherapies of Alzheimer's Disease
}

\begin{abstract}
Jia-yan Xin ${ }^{1,2}$, Xiao-yan Zhu ${ }^{3}$, Xiao Huang ${ }^{1,2}$, Yu-hui Liu ${ }^{4}$, Jun Tan $^{5}$ and Yang Xiang ${ }^{6,7 *}$
${ }^{1}$ Department of Clinical Medicine, North Sichuan Medical College, Nanchong, China, ${ }^{2}$ Department of Neurology, General Hospital of Western Theater Command, Chengdu, China, ${ }^{3}$ Basic Medical Laboratory, General Hospital of Western Theater Command, Chengdu, China, ${ }^{4}$ Department of Neurology and Centre for Clinical Neuroscience, Daping Hospital, Third Military Medical University, Chongqing, China, ${ }^{5}$ Department of Obstetrics and Gynecology, Affiliated Hospital of Guizhou Medical University, Guiyang, China, ${ }^{6}$ Department of Neurology, Sichuan Provincial People's Hospital, University of Electronic Science and Technology of China, Chengdu, China, ${ }^{7}$ Chinese Academy of Sciences Sichuan Translational Medicine Research Hospital, Chengdu, China
\end{abstract}

Keywords: immunological mechanism, biomarker, immunotherapy, Alzheimer's disease, peripheral system

\section{Editorial on the Research Topic}

Immunological Mechanisms, Biomarkers, and Immunotherapies of Alzheimer's Disease

Alzheimer's disease is the most common type of dementia characterized by neuropathological changes such as intracellular tau tangles and extracellular deposition of $\beta$-amyloid $(\mathrm{A} \beta)$ as plaques. At present, the study of pathogenesis, the search for biomarkers, and the development of treatment strategies of $\mathrm{AD}$ are all closely related to immunological theory and techniques. The main aim of the current Research Topic was to provide a current collection of immunological mechanisms, biomarkers, and therapeutic strategies in $\mathrm{AD}$.

Growing evidence has proved that Alzheimer's disease (AD), as a typical degenerative disease of the central nervous system, has complex connections with the peripheral system (Wang et al., 2017), such as the peripheral clearance of pathogenic substances ( $A \beta$ and Tau) or the impact of gut microbiota on AD-like pathological characteristics, etc. Among them, the relationship between systemic autoimmune diseases and $\mathrm{AD}$ has been puzzling researchers for years. Culibrk and Hahn summarized the link of chronic inflammatory osteoarthritis disease with late-onset AD. In particular, they focused on the pathophysiological characteristics and immunotherapy of rheumatoid arthritis, osteoarthritis, and osteoporosis, as well as their implications on AD pathogenesis. They also summarized some other possible mechanisms, including the agerelated cellular/immuno-senescence, the aberrant peripheral nervous system activity, dysregulated autophagic homeostasis, and the pathological microRNA profiles, which could attract more attention from the audience.

The relation between hereditary amyloidosis and $\mathrm{AD}$ is a topic rarely discussed before. Although the pathogenic substances of the two diseases are different, it is not an obstacle to investigating their association from the perspective of genetics. Jiang et al. reported two novel likely pathogenic frame-shift mutations of gelsolin in $\mathrm{AD}$ patients using the genes targeted sequencing (GTS) method. Intriguingly, both mutations seem to correlate with the age at which clinical symptoms of $\mathrm{AD}$ appeared, respectively, even though the gelsolin is a common pathogenic gene charged with hereditary amyloidosis.

The development of biomarkers is a spotlight in $\mathrm{AD}$ research over the past decade. The proposal of the NIA-AA research framework of AD in 2018 not only categorizes AD biomarkers as per the 
AT(N) system but also provides more objective and rigid biological criteria for the diagnosis of $\mathrm{AD}$ than ever before (Jack et al., 2018). Since the presence or absence of $A \beta$ is the sole criterium for the etiologic diagnosis of $A D$ in the $A T(N)$ system, the emerging novel non- $\mathrm{A} \beta$ biomarkers are mostly classified in the $(\mathrm{N})$ catalog. Based on the Alzheimer's Disease Neuroimaging Initiative (ADNI) cohort, Pillai, Bebek et al. measured the levels of tumor necrosis factor receptor (TNFR) 2 in the cerebrospinal fluid (CSF) and investigated its correlations with CSF t-Tau, CSF p-Tau, the cognitive domains, the MRI measures, and the longitudinal cognitive changes, together indicating the potential value of TNFR2 in the diagnosis and treatment of AD.

The biomarker research has gradually shifted from CSF to blood due to the invasive operation, difficult acquisition, and patient unacceptability of CSF sampling (Zetterberg and Blennow, 2018). Wang J. et al. compared the naturally occurring antibody to $\alpha$-synuclein (NAb-a-Syn) in the blood of patients with Parkinson's disease dementia, $\mathrm{AD}$, vascular dementia, and normal controls, and further explored the correlations between NAb-a-Syn and the severity of cognitive impairment and PD, respectively. Of note, the naturally occurring antibodies also have a good performance in the immunotherapy of AD (Liu et al., 2021).

As a shining star of $\mathrm{AD}$ biomarkers in the past years, the value of neurofilament light chain (NfL) in $\mathrm{AD}$ diagnosis and prognosis is no longer needed to be described. Especially, the blood-NfL is consistent and comparable with CSF-NfL, which greatly increases the clinical application value of NfL (Fortea et al., 2018). Wang $\mathrm{J}-\mathrm{H}$. et al. evaluated the difference of blood-NfL in patients with post-stroke subjective cognitive decline (SCD). Although this study was a single-center prospective study with a small sample size, post-stroke SCD is a clinical condition that has received little attention before, showing the careful observation and thinking of the authors.

Likewise, neurogranin has emerged as an important biomarker for neurodegenerative diseases in recent years, although the current studies have not demonstrated that its clinical value is comparable to that of the NfL. Xiang et al. reviewed the associations between neurogranin and neurological and psychiatric diseases and further made the perspectives on the research and applications of neurogranin in the future.

Compared with body fluid-based biomarkers, image-based biomarkers have not developed rapidly over recent decades. The clinical applications of $\mathrm{A} \beta$-positron emission tomography (PET) and tau-PET have promoted the early diagnosis and severity assessment of AD (Hansson, 2021); however, the high cost and low coverage of PET limit its clinical application. Many reports on functional magnetic resonance imaging (fMRI) emerged, but fMRI has not yet reached the same value as that of A $\beta$-PET and tau-PET in the diagnosis of AD. Based on ADNI, Yang et al.

\section{REFERENCES}

Fortea, J., Carmona-Iragui, M., Benejam, B., Fernandez, S., Videla, L., Barroeta, I., et al. (2018). Plasma and CSF biomarkers for the diagnosis of Alzheimer's disease in adults with Down syndrome: a cross-sectional study. Lancet Neurol. 17, 860-869. doi: 10.1016/S1474-4422(18)30285-0 studied the dynamics and concordance changes in different brain regions in the patients with SCD using resting-state fMRI (RSfMRI), adding the new evidence for the use of $\mathrm{PMRI}$ in AD.

Researchers have long been interested in acupuncture for the treatment of neurological diseases, even though its mechanism is not fully understood (Kaptchuk, 2002). Based on growing published data, Huang et al. analyzed the therapeutic effect of acupuncture on AD using a meta-analysis method. The authors proposed that high-quality studies with rigorous study designs and larger samples are required in the future, even though it is now generally believed that acupuncture is a promising complementary treatment for AD.

Over the past decades, a variety of hypotheses about the mechanism of $\mathrm{AD}$ have emerged. At present, even the amyloid cascade hypothesis either have to face updates or have to face challenges (Tolar et al., 2020). A growing body of evidence suggests that the mechanisms of $\mathrm{AD}$ beyond $\mathrm{A} \beta$ and Tau are possible in which the neurovascular unit, brain lymphatic system, gut microbiota, and neuroinflammation could be involved (Henstridge et al., 2019). Among them, the activation of the immune system is the key regulatory factor of $\mathrm{AD}$ pathology (Long and Holtzman, 2019). Moreover, almost all the clinical trials of anti-AD drugs targeting $\mathrm{A} \beta$ or Tau failed or have been inconclusive (Mullard, 2021), suggesting that the immunotherapy strategies may also need to concentrate on some other targets rather than just $A \beta$ and Tau.

We have always regarded $\mathrm{AD}$ as a degenerative disease of the central nervous system. However, more and more evidence confirms that the peripheral system has a great influence on AD. As an essential part of the peripheral system, the role played by the immune system and tissues and organs rich in immune cells in the pathogenesis of $\mathrm{AD}$ have not been fully answered. The spleen, intestine, omentum, and blood, these seemingly distant and unrelated organs and tissues are likely to affect our brain structure and cognitive function via immunological regulation, fascinating scientific issues for further and future research.

\section{AUTHOR CONTRIBUTIONS}

All authors listed have made a substantial, direct and intellectual contribution to the work, and approved it for publication.

\section{FUNDING}

This study was supported by the National Natural Science Foundation of China (81601112, 81801090), Top Project of the Youth Incubation Program of Military Medical Science and Technology (19QNP065), and the Sichuan Department of Science and Technology Fund (2018SZ0141, 2019YSF0213).

Hansson, O. (2021). Biomarkers for neurodegenerative diseases. Nat. Med. 27, 954-963. doi: 10.1038/s41591-021-01 382-x

Henstridge, C. M., Hyman, B. T., and Spires-Jones, T. L. (2019). Beyond the neuron-cellular interactions early in Alzheimer disease pathogenesis. Nat. Rev. Neurosci. 20, 94-108. doi: 10.1038/s41583-018-0113-1 
Jack, C. R. Jr., Bennett, D. A., Blennow, K., Carrillo, M. C., Dunn, B., Haeberlein, S. B., et al. (2018). NIA-AA Research Framework: toward a biological definition of Alzheimer's disease. Alzheimers Dement. 14, 535-562. doi: 10.1016/j.jalz.2018.02.018

Kaptchuk, T. J. (2002). Acupuncture: theory, efficacy, and practice. Ann. Intern. Med. 136, 374-383. doi: 10.7326/0003-4819-136-5-200203050-00010

Liu, Y. H., Wang, J., Li, Q. X., Fowler, C. J., Zeng, F., Deng, J., et al. (2021). Association of naturally occurring antibodies to beta-amyloid with cognitive decline and cerebral amyloidosis in Alzheimer's disease. Sci. Adv. 7:abb0457. doi: 10.1126/sciadv.abb0457

Long, J. M., and Holtzman, D. M. (2019). Alzheimer disease: an update on pathobiology and treatment strategies. Cell 179, 312-339. doi: 10.1016/j.cell.2019.09.001

Mullard, A. (2021). Landmark Alzheimer's drug approval confounds research community. Nature 594, 309-310. doi: 10.1038/d41586-021-01546-2

Tolar, M., Abushakra, S., and Sabbagh, M. (2020). The path forward in Alzheimer's disease therapeutics: reevaluating the amyloid cascade hypothesis. Alzheimers Dement. 16, 1553-1560. doi: 10.1016/j.jalz.2019.09.075

Wang, J., Gu, B. J., Masters, C. L., and Wang, Y. J. (2017). A systemic view of Alzheimer disease - insights from amyloid-beta metabolism beyond the brain. Nat. Rev. Neurol. 13, 612-623. doi: 10.1038/nrneurol.201 7.111
Zetterberg, H., and Blennow, K. (2018). From cerebrospinal fluid to blood: the third wave of fluid biomarkers for Alzheimer's disease. J. Alzheimers Dis. 64, S271-S279. doi: 10.3233/JAD-179926

Conflict of Interest: The authors declare that the research was conducted in the absence of any commercial or financial relationships that could be construed as a potential conflict of interest.

Publisher's Note: All claims expressed in this article are solely those of the authors and do not necessarily represent those of their affiliated organizations, or those of the publisher, the editors and the reviewers. Any product that may be evaluated in this article, or claim that may be made by its manufacturer, is not guaranteed or endorsed by the publisher.

Copyright (๑) 2021 Xin, Zhu, Huang, Liu, Tan and Xiang. This is an open-access article distributed under the terms of the Creative Commons Attribution License (CC $B Y)$. The use, distribution or reproduction in other forums is permitted, provided the original author(s) and the copyright owner(s) are credited and that the original publication in this journal is cited, in accordance with accepted academic practice. No use, distribution or reproduction is permitted which does not comply with these terms. 